\title{
3D bioprinting-a step towards heart tissue regeneration
}

\begin{abstract}
Heart disease and cardiovascular disease is a very serious and growing public health issue. Tissue-engineering has great potential and great strength for regeneration, remolding, and growth. In the case of heart failure, Allografting has been used. 3D bioprinting has a great impact in the field of cardiovascular tissue engineering. It has been observed that 3D Bioprinting is used to construct an artificial heart for transplantation and used to create myocardial cells in case of injury. Recent studies showed that biomaterial used in the treatment of myocardial dysfunction is decellularized cardiac extracellular matrix hydrogel in adults. Collagen, Alginate gelatin, hyaluronic acid, and deECM scaffolds were used as biomaterials in 3D bioprinting. It has been shown that scaffold used with ECM was used to support there generation process. A new 3D bioprinting technology was developed in which cells were collected into spheroids and printed on a needle array according to desirable characteristics. Different bio inks such as laser, extrusion, droplet, and stereolithography are used here. Electric stimulation is key to the contractility of cardiomyocytes. A physical cardiac replica was created by image processing software that creates $3 \mathrm{D}$ structures. In holographic display $3 \mathrm{D}$, full hearts of patients were printed in flexible material. A process is demonstrated to fabricate robust valves of the heart using the3D bioprinting technique. MRI or CT scans were used to obtained 3D images of the aorta.3D bioprinting plays a huge role in knowing the aortic anatomy involves the aortic valve area and morphology of the root. Recent advances demonstrated that $3 \mathrm{D}$ bioprinting can assist in ventricular device placement and perform a specific function in a complex with (CHD) Congenital heart defects. 3D bioprinting holds great promise towards patient-tailored cardiovascular practice, and in clinical tool development.
\end{abstract}

Keywords: myocardial cells, transplantation, bioprinting, biomaterial
Volume 8 Issue I - 202 I

\author{
Sana Shahzadi, Isha Ishtiaq, Khadija Aslam, \\ Usama Ali, Savera Mehak, Sara Khan, Shanza \\ Sajjad, Maria Babar
}

Department of Biotechnology, University of Sialkot, Pakistan

Correspondence: Sana Shahzadi, Department of Biotechnology, University of Sialkot, Sialkot, Pakistan, Email sanaamughal034@gmail.com

Received: November 21, 2020 | Published: January 07, 2021
Abbreviations: CHD, congenital heart defects; CVD, cardiovascular disease; CT, computer tomography

\section{Introduction}

Cardiovascular disease(CVD) is the leading cause of death worldwide.35,000 newborns are affected by congenital heart diseases per year. ${ }^{1-5}$ The problem in blood arteries or electrical impulses causes a heart attack or cardiac arrest because they supply nutrients and oxygen to heart tissues. ${ }^{6-8}$ Organ transplantation i.e. allografting has been used in case of heart failure but it has a limitation that there is often less organ availability and immune rejection. ${ }^{4,7}$ Cardiovascular tissue engineering is used to repair heart valves and cardiac damage. ${ }^{4}$ To study heart valves interstitial cell response PEG-based hydrogels have been used. ${ }^{9-11}$ It was studied that the function of the ventricular heart can be improved by replacing necrotic tissue with tissueengineered construct and it is known as heart patch. Heart patches are formed by hydrogels, synthetic polymers, and de-cellularized extracellular matrix. ${ }^{6} 3 \mathrm{D}$ bioprinting has a great impact in the field of cardiovascular engineering. It is a technique that forms 3D structures and it is fully assisting on computer-aided design (CAD). Patientspecific models have been created by $3 \mathrm{D}$ printing techniques. ${ }^{9}$ Recent studies showed that biomaterial used in the treatment of myocardial dysfunction is decellularized cardiac extracellular matrix hydrogel in adults. ${ }^{5}$ Alginate, collagen, gelatin, hyaluronic acid, and deECM scaffolds were used as biomaterials in 3D bioprinting. Two types of techniques: scaffold-based and scaffold-free were used to get desirable $3 \mathrm{D}$ constructs. It was investigated that both these techniques have certain limitations. It has been observed that a scaffold with ECM material was used to support the regeneration process. ${ }^{8}$ The main purpose is to adopt $3 \mathrm{D}$ printing as a fabrication strategy for tissue engineering of heart valves and some problems occur in the classical assembly of scaffold and they want to overcome it. ${ }^{10}$ It has been observed that decellularized tissues have also been used as scaffolds. The benefit of using decellularized tissues is that their structure is similar to natural tissues. ${ }^{13}$ A new3D bioprinting technology was developed in which cells were collected into spheroids and printed on a needle array according to desirable characteristics. A scaffold-free tubular construct was fabricated. ${ }^{7}$ Without the need for postoperative medications, terminal heart failure would get their new hearts from their cells. Valvular conduits was formed by GELMA based hydrogels technique. ${ }^{12}$ It has been observed that valvular mechanobiology could give high throughput and performance in CAVD. ${ }^{12}$ Imaging technologies such as Computed Tomography (CT),Magnetic resonance imaging are used to draw the structural information of native tissues and then created $3 \mathrm{D}$ printable files. ${ }^{9}$ Severe heart diseases and human tissue model production was treated by Cell sheet based tissue engineering because it has showed a great potential to fabricate 3D cardiac tissues. ${ }^{13}$ Electric stimulation is a key factor in contractility of cardiomyocytes. ${ }^{14-21}$ Physical cardiac replica was created by image processing software that creates a 3D structures. In holographic display 3D full hearts of patients were printed in flexible material. ${ }^{4}$ In recent studies using pulse rate retrograde aortic perfusion adult porcine heart was successfully decellularized less than 10 hours. ${ }^{17}$ Recent advances showed that 3D bioprinting can work with ventricular based device placement and perform a function in(CHD) Congenital heart defects. With complex CHD, 3Dbioprinting can also help with transplant planning for recipients. ${ }^{15}$ 
Table I Different materials used in3D printing of cardiac structures

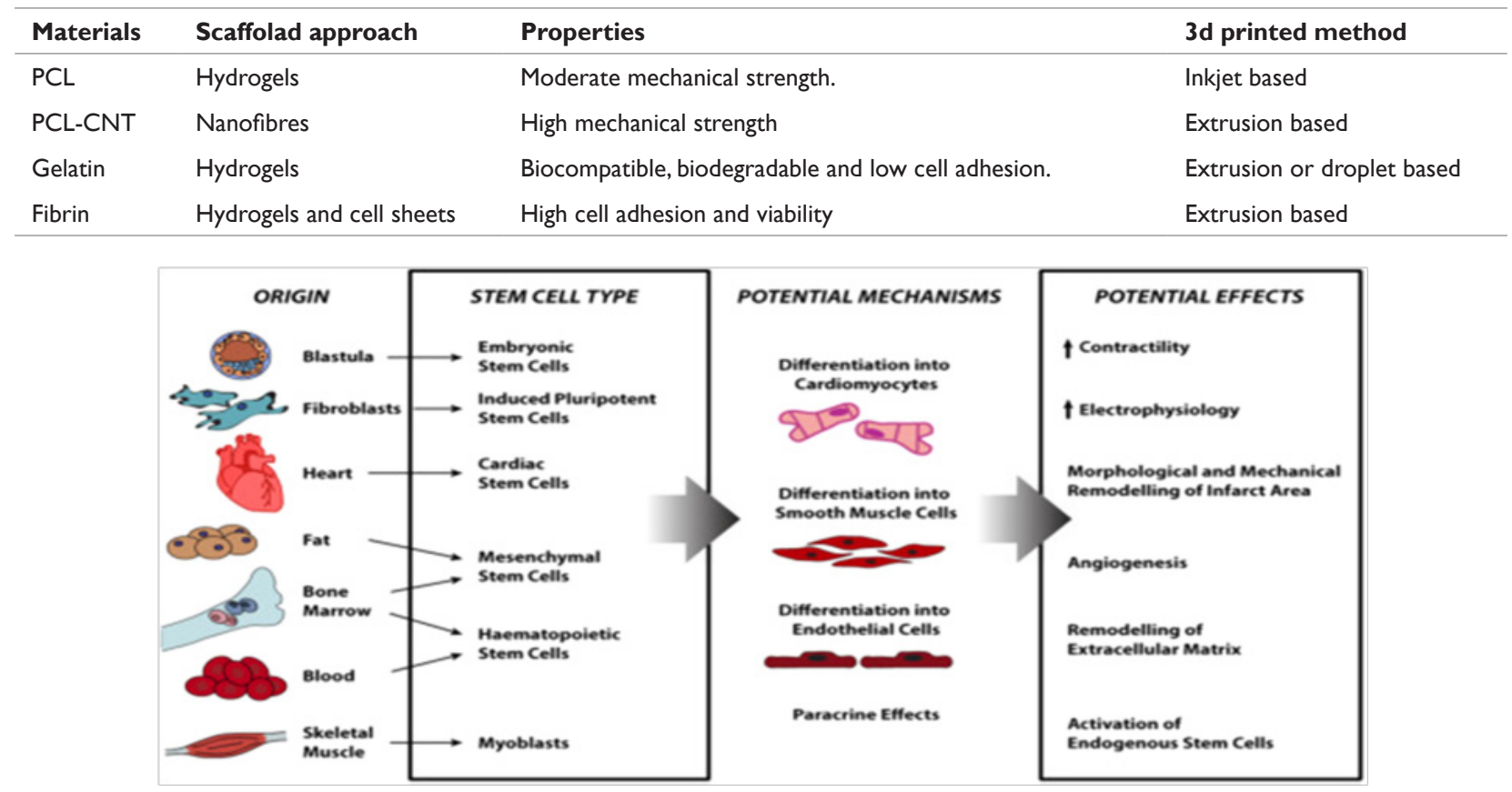

Figure I Cells for cardiac tissue engineering.

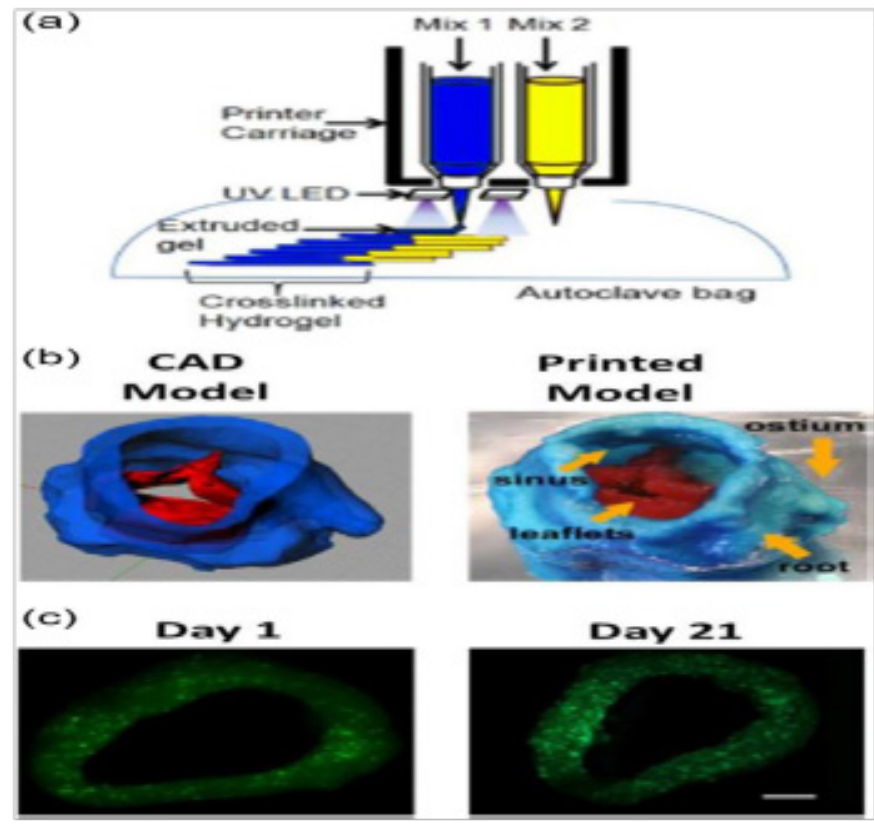

Figure 2 3D bioprinted of heart (a)3D printer method include two extrusion system with Ultraviolent light source(b)CAD Model. Red area indicates leaflets and blue area indicates heart valve (c)Microscopic images of PAVIC cell.

\section{Modes of bioprinting}

Bioinks process several properties such as biocompatibility, printability, and biomimicry. ${ }^{16}$ Extrusion-based, laser-based, droplet based and sterolithograohy are bioprinting processes that are used here. Injection moulding is also another 3D bioprinting technology. There are different materials used for medical 3D printing from resin to nylon and metals.

\section{Extrusion based \\ 2. Laser based \\ 3. Sterolithography \\ 4. Inkjet bioprinting}

\section{Extrusion-based bioprinting}

Extrusion bioprinting is followed by viscoelastic biomaterial expulsion and they are called bioinks then they form 3D construct in layer by layer method. The nozzle that used in this process dispense the bioink and they move in 3 directions and at that stage printer can be set. When increase the nozzles's diameter it can produce thicker filaments as a result stable structures occurs. ${ }^{4}$ The most popular extrusion based method is FRESH. Materials of low viscosity used in $3 \mathrm{~d}$ structures. ${ }^{2}$ Extrusion based bioprinting process is in moderate cost, produce low resolution, and it is very low technique.

\section{Laser-based bioprinting}

This technique has been used to form in vitro constructs and linked with different properties including. large cell densities, increased cellviability, highresolution, printing speed high. To form the desired tissue structures this technique used 2 slides namely an upper and other lower slide. This method is also known as LIFT. By stacking biomaterials droplets LIFT provides both $2 \mathrm{D}$ and 3D models. ${ }^{4}$

\section{Stereolithography}

This technique is used to treat bioink resins in a plane by plane direction by the digital light projector. Stereolithiography uses popular hydrogel such as polyethylene glycol diacrylate. Using stereolithographic bioprinting hydrogel and cell laden have also been created. By using (MRI) Magnetic reasonance imaging or (CT) Computer Tomography scan 3D structure images of the aorta were obtained. ${ }^{2}$ This technique is only best for photopolymerizable bioinks. ${ }^{9}$ 


\section{Inkjet bioprinting}

This inkjet bioprinter is the same as a commercial desktop inkjet bioprinter. This technique is mostly used for materials that have low viscosity that is collagen and fibrin. Due to its high speed, this technique is not good to fabricate structures in a vertical plane This inkjet technique is not used to fabricate TEVGs. ${ }^{2}$

\section{D printing for heart valves}

There are two methods available for replacement therapy of heart valves. Usage of a heart valve which is mechanical and usage of a heart valve which is biological. The main benefit of prosthetic valves is that they are robust, and they have a long lifetime. The disadvantage of valves is that to take an anticoagulant for a life they require patient. Allographic or xenographic sources are used to make biological valves. Biological valves do not require the patient for anticoagulants but they have a shorter life than prosthetic valves. Tissue-engineered heart valves use their patient cell, improve hemocapability of valves. For any heart quality desired qualities are given below

1) They have a low-pressure gradient for transvalvular

2) To repair the damage they have a high capacity

3) They have a low thrombogenic response., ${ }^{1,2}$

Recently Jonathon butcher labs perform the process of 3D bioprinting of tissue-engineered heart valves. In2012 Bucher team developed another 3D bioprinting process which is based on extrusionbased. It is based on two types of photo cross linkable hydrogels one hydrogel is rigid which is best for the root, and the other is soft which is used for leaflets. In 45 minutes the heart was $3 \mathrm{D}$ printed. It has been demonstrated that porcine artificial interstitial cells can be inserted in to the heart valve of 3D printed. Most Recently Butcher team observed that Methacrylated hydrogels are used with human aortic valvular interstitial cell (HAVIC) IN 3D bioprinting. The HAVIC cells can remold the hydrogels with their own ECM. ${ }^{1}$ It has been shown by the Butcher team 3D bioprinting is best for valves of the heart because

1) They have complex geometry

2) They require heterogeneity

3) They have a low contribution of activity.

\section{Applications of $3 \mathrm{~d}$ bioprinting in CVDS}

$3 \mathrm{~d}$ bioprinting is an emerging field. ${ }^{23}$ 3Dbioprintingplays very important role in knowing heart disease, cardiac masses, and bioprinting.3D bioprinting has been used in treatment and diagnosis of heart valves. $3 \mathrm{~d}$ bioprinting also helps in understanding the anatomy of aortic area and morphology of root. $^{8}$ 3Dbioprinting also helps in the surgical procedures in case of septal myectomy. 3Dstructure heart model is very helpful and useful for congenital disease.3D bioprinting also helps in communication with the patients.3Dmodels are also use in intensive care unit (ICU)to explain treatment plans.3D printed also have main application in treatment of cardiovascular disease. They are used to enhance the spatial relationship of congenital diseases.3D bioprinting are used to construct artificial heart for transplantation.3D bioprinting is used to construct Myocardial cells in the case of injury. For operative procedures 3D model increases the confidence level of specialist. ${ }^{8}$ AUV integrated 3D bioprinting technique was used to create hybrid hydrogel constructs and it consists of Carbon nano tubes emerged framework and Cell laden MeCOL. ${ }^{19}$

\section{Conclusion}

3D bioprinting holds great promise towards patient-tailored cardiovascular practice, and in clinical tool development.3D printing also helps in the education of patients and physicians.3D bioprinted cell encapsulated valve conduits and they have great potential for tissue-engineered living valve replacement. 3D printing is adopted as a fabrication strategy for tissue engineering of heart valves, and some problems that occur in the classical assembly of a scaffold, and want to overcome them. It is demonstrated that a process to fabricate robust heart valves using a 3Dprinting technique. MRI or CT scans were used to obtained 3D images of the aorta. Electrical stimulation is key to cardiomyocyte contractility. FRESH is the most popular extrusionbased method. It has been observed that a scaffold with ECM material was used to support the regeneration process. Recent advances showed that 3Dbioprintingcould help in ventricular assist device placement and perform a function in CHD. With complex CHD, 3Dbioprinting can also assist with transplant planning for recipients.

\section{Acknowledgements}

None.

\section{Conflicts of interest}

No conflict of interest was reported by the authors.

\section{Funding}

None.

\section{References}

1. Bobak Mosadegh, Guanglei Xiong, Simon Dunham, et al. Current progress in 3D printing for cardiovascular tissue engineering. Biomedical Materials. 2015;10(3):034002.

2. Lee Wenhan, Yi Hong, Guohao Dai. 3D bioprinting of vascular conduits for pediatric congenital heart repairs. Translational Research 2019.

3. Rajat Vashistha, Prasoon Kumar, Arun Kumar Dangi, et al. Quest for cardiovascular interventions: precise modeling and 3D printing of heart valves. Journal of biological engineering. 2019;13(1):12.

4. Matthew Alonzo, Shweta Anil Kumar, Brian Roman, et al. 3D Bioprinting of cardiac tissue and cardiac stem cell therapy. Translational Research. 2019.

5. Donald Bejleri, Benjamin W Streeter, Aline L Y Nachlas, et al. A Bio printed Cardiac Patch Composed of Cardiac-Specific Extracellular Matrix and Progenitor Cells for Heart Repair. Advanced healthcare materials. 2018;7(23):1800672.

6. Ajay Tijore, Scott Alexander Irvine, Udi Sarig, et al. Contact guidance for cardiac tissue engineering using 3D bioprinted gelatin patterned hydrogel. Biofabrication. 2018;10(2):025003.

7. Kenichi Arai,Daiki Murata, Ana Raquel Verissimo, et al. Fabrication of scaffold-free tubular cardiac constructs using a Bio-3D printer. PloS one. 2018;13(2):0209162.

8. Qasim Muhammad, Haq F, Kang MH, et al. 3D printing approaches for cardiac tissue engineering and role of immune modulation in tissue regeneration. International journal of nanomedicine. 2019;14:1311.

9. $3 \mathrm{D}$ bioprinting for cardiovascular regeneration and pharmacology.

10. Laura Ann Hockaday, Bin Duan, Kevin Heeyong Kang, et al. 3D-printed hydrogel technologies for tissue-engineered heart valves. 3D Printing and Additive Manufacturing. 2014;1(3):122-136. 
11. Laura Hockaday Kang, Patrick Armstrong, Lauren Lee, et al Optimizing photo-encapsulation viability of heart valve cell types in 3D printable composite hydrogels. Annals of biomedical engineering. 2017;45(2):360-377.

12. Dewy C van der Valk, Casper F T van der Ven, Mark C Blaser, et al Engineering a 3D-bioprinted model of human heart valve disease using nanoindentation-based biomechanics. Nanomaterials. 2018;8(5):296.

13. Jang Jinah. 3D bioprinting and in vitro cardiovascular tissue modeling. Bioengineering. 2017;4(3):71

14. John M Wainwright, Caitlin A Czajka, Urvi B Patel, et al. Preparation of cardiac extracellular matrix from an intact porcine heart. Tissue Engineering Part C: Methods. 2009;16(3):525-532.

15. Taylor DA, LC Sampaio, A Gobin. Building new hearts: a review of trends in cardiac tissue engineering. American Journal of Transplantation. 2014;14(11):2448-2459.

16. Mohammad Izadifar, Dean Chapman, Paul Babyn, et al. UV-assisted 3D bioprinting of nanoreinforced hybrid cardiac patch for myocardial tissue engineering. Tissue Engineering Part C: Methods. 2018;24(20):74-88.
17. Maria Kitsara, Dimitrios Kontziampasis, Onnik Agbulut, et al. Heart on a chip: micro-nanofabrication and microfluidics steering the future of cardiac tissue engineering. Microelectronic Engineering. 2019;203:44 622 .

18. Cristina Colosi, Su Ryon Shin, Vijayan Manoharan, et al. Microfluidic bioprinting of heterogeneous 3D tissue constructs using low-viscosity bioink. Advanced materials. 2016;28(4).

19. Thomas A Foley, Abdallah El Sabbagh, Nandan S Anavekar, et al. 3D-printing: applications in cardiovascular imaging. Current Radiology Reports. 2017;5(9).

20. Gao Q, Liu Z, Lin Z, et al. 3D bioprinting of vessel-like structures with multilevel fluidic channels. ACS biomaterials science \& engineering. 2017;3(3):399-408.

21. Farooqi KM, Cooper C, Chelliah A, et al. 3d printing and heart failure: The present and the future. JACC: Heart Failure. 2018. 